\title{
Effect of kiwifruit on metabolic health in patients with cardiovascular risk factors: a systematic review and meta-analysis
}

This article was published in the following Dove Medical Press journal: Diabetes, Metabolic Syndrome and Obesity:Targets and Therapy

\author{
Naeti Suksomboon' \\ Nalinee Poolsup ${ }^{2}$ \\ Wei Lin' \\ 'Department of Pharmacy, Faculty \\ of Pharmacy, Mahidol University, \\ Bangkok, Thailand; '2Department \\ of Pharmacy, Faculty of Pharmacy, \\ Silpakorn University, Nakhon Pathom, \\ Thailand
}

Correspondence: Nalinee Poolsup Department of Pharmacy, Faculty of Pharmacy, Silpakorn University, 6 Rajamankha Nai Road, Amphoe Muang, Nakhon Pathom, 73000, Thailand Email npoolsup@hotmail.com
Background: Kiwifruit seems to have beneficial effect on metabolic health because it contains abundant phytochemicals and antioxidants. This study aimed to assess the effect of kiwifruit on metabolic health in participants with cardiovascular risk factors.

Methods: Literature was searched from PubMed, CENTRAL, Cumulative Index to Nursing and Allied Health Literature, Web of Science, Scopus, Proquest, Latin American and Caribbean Health Sciences Literature, International Clinical Trials Registry Platform, Australia New Zealand Clinical Trials Registry, https://clinicaltrials.gov/, China National Knowledge Infrastructure, Wanfang Standards Database, European Association for the Study of Diabetes, and American Diabetes Association conferences up to August 2018. Citing references were manually searched. Randomized controlled trials were selected if they evaluated the effect of kiwifruit in patients with cardiovascular risk factors and reported SBP, DBP, total cholesterol (TC), triglyceride (TG), high-density lipoprotein cholesterol (HDL-C), low-density lipoprotein cholesterol (LDL-C), glycated hemoglobin (A1C), fasting plasma glucose (FPG), homeostasis model assessment of insulin resistance (HOMA-IR), 2-hour postprandial glucose, or body weight (BW). Data extraction and study quality assessment were performed independently by two investigators. Any inconsistencies were resolved by a third investigator. Treatment effect was estimated with mean difference (MD). Effect estimates were pooled using inverse-variance weighted method. Heterogeneity was assessed by the $I^{2}$ and $Q$ statistic.

Results: Five randomized controlled trials involving 489 participants met the inclusion criteria. These included hypercholesterolemia, hypertension, type 2 diabetes mellitus, and male smokers. There was no effect of kiwifruit on SBP (MD, $-1.72 \mathrm{mmHg}$; 95\% CI: -4.27 to 0.84 ); DBP (MD, $-2.35 \mathrm{mmHg}$; 95\% CI: -5.10 to 0.41 ); TC (MD, $-0.14 \mathrm{mmol} / \mathrm{L} ; 95 \% \mathrm{CI}:-0.71$ to 0.43 ); TG (MD, $-0.23 \mathrm{mmol} / \mathrm{L} ; 95 \% \mathrm{CI}:-0.66$ to 0.20$)$; LDL-C (MD, $-0.41 \mathrm{mmol} / \mathrm{L} ; 95 \% \mathrm{CI}:-0.99$ to 0.18 ); HDL-C (MD, $0.15 \mathrm{mmol} / \mathrm{L}$; $95 \% \mathrm{CI}:-0.18$ to 0.48$)$; FPG (MD, $-0.08 \mathrm{mmol} / \mathrm{L} ; 95 \%$ CI: -0.37 to 0.21 ); HOMA-IR (MD, -0.29 ; $95 \% \mathrm{CI}:-0.61$ to 0.02 ), and BW (MD, $-1.08 \mathrm{~kg}$; 95\% CI: -4.22 to 2.05 ).

Conclusion: This meta-analysis suggested no effect of kiwifruit on metabolic health in patients with cardiovascular risk factors, although there seemed to be a trend of improvement after kiwifruit intervention

Keywords: Actinidia, metabolic diseases, lipid, cholesterol, blood glucose, blood pressure

\section{Introduction}

Metabolic health has been defined in many different ways. Metabolic abnormalities include obesity, dyslipidemia, hypertension, insulin resistance, and proinflammatory 
status. ${ }^{1}$ There were $\sim 83.5$ million cases of cardiovascular disease (CVD) in European countries in 2017, and there were around 11 million new cases that increased steadily compared to $1990 .^{2}$ The risk factors of CVD are nonmodifiable and modifiable. Nonmodifiable risk factors include increased age, male sex, and family history of CVD. Modifiable risk factors consist of high cholesterol, high low-density lipoprotein (LDL) and triglycerides (TGs), high blood pressure, diabetes, smoking, obesity, and excessive alcohol consumption. ${ }^{3,4}$ There are currently several varieties of medications being prescribed for metabolic disease management. However, pharmacotherapy not only exerts positive effects but also brings unexpected adverse effects. Therefore, attempts have been made to find alternative management, such as fruit products, to help patients deal with metabolic problems.

Kiwifruit is also known as "mihoutao" in Chinese or Actinidia. It is a member of Ericales order, Actinidiaceae family, genus Actinidia. ${ }^{5}$ The genus Actinidia is variable, containing around 60 species. ${ }^{6,7}$ Kiwifruit contains fat, carbohydrates (sugar and dietary fiber), protein (lutein and zeaxanthin), vitamins A, B, C, E, and K, minerals, flavonoid, polyphenols, inositol, and carotenoids. Being rich in vitamins and antioxidants, it seems helpful for metabolic health. Some animal studies have tested the effect of kiwifruit on blood glucose. For example, a 1-week study was conducted in 30 wistar male rats. ${ }^{8}$ Rats were divided into five groups (six rats per group [G], G1: nondiabetic control, G2: alloxan-induced diabetic control, G3: diabetic rats given Actinidia deliciosa extract $500 \mathrm{mg} / \mathrm{kg}, \mathrm{G} 4$ : diabetic rat given A. deliciosa extract $1,000 \mathrm{mg} / \mathrm{kg}$, and G5: diabetic rats given metformin $10 \mathrm{mg} /$ $\mathrm{kg}$ ). Both concentrations of $A$. deliciosa fruit extract were reported to decrease blood glucose level significantly compared to diabetic control group (G3: $186 \mathrm{mg} / \mathrm{dL}, \mathrm{G} 4: 151.2$ $\mathrm{mg} / \mathrm{dL}$ vs G2: $269 \mathrm{mg} / \mathrm{dL}, P<0.001) .{ }^{8}$ A clinical study of kiwifruit intervention was performed in hyperlipidemic patients. ${ }^{9}$ Participants consumed two kiwifruits (100 g each) per day for 8 weeks and were found to have significant increase in high-density lipoprotein-cholesterol level compared to baseline. However, no significant differences were detected between baseline and final assessment for triacylglycerol, total cholesterol (TC), and LDL cholesterol. Another randomized crossover study was aimed to assess whether kiwifruit decreased platelet activity and lipid profile in healthy volunteers. ${ }^{10}$ It was reported that consumption of two or three kiwifruits per day for 28 days significantly reduced TG level by $15 \%$ compared to control group. No effect was detected with cholesterol levels. Several other trials were conducted to evaluate the effects of kiwifruit on metabolic health. We, therefore, performed a systematic review and meta-analysis in an attempt to assess the effect of kiwifruit on metabolic parameters in participants with cardiovascular risk factors.

\section{Methods}

A systemic review and meta-analysis was conducted following PRISMA guideline. ${ }^{11}$

\section{Data sources}

Studies that assessed the effect of kiwifruit on metabolic health in patients with cardiovascular risk factors were selected. Literature search was conducted from the respective inception until August 2018 without language restriction. The following databases were searched: PubMed, CENTRAL, Cumulative Index to Nursing and Allied Health Literature, Web of Science, Scopus, Proquest, Latin American and Caribbean Health Sciences Literature, International Clinical Trials Registry Platform, Australia New Zealand Clinical Trials Registry, https:// clinicaltrials.gov/, China National Knowledge Infrastructure, and Wanfang Standards Database. European Association for the Study of Diabetes and American Diabetes Association conferences were also scanned. The historical search of related citations was conducted. Non-English language literatures were translated into English. Search strategy was performed using medical subject headings (MeSH) terms: Actinidia, metabolic diseases, diabetes mellitus, cholesterol, lipids, dyslipidemias, hypertension, blood pressure, blood glucose. This was followed by keyword search using the following keywords: kiwifruit, Actinidia chinensis, Actinidia deliciosa, Actinidia arguta, Actinidia kolomikta, fasting plasma glucose, 2-hour postprandial glucose, and A1C.

\section{Selection criteria}

Studies were included in the systematic review if they were 1) randomized controlled trials that evaluated the effect of kiwifruit in participants with cardiovascular risk factors, including hypertension, dyslipidemia, type 2 diabetes mellitus (T2DM), and smoking, and 2) reporting outcomes containing SBP, DBP, glycated hemoglobin (A1C), fasting plasma glucose (FPG), 2-hour postprandial glucose (2-h PG), body weight $(\mathrm{BW})$, homeostasis model assessment of insulin resistance (HOMA-IR), TC, TG, HDL, or LDL.

\section{Data extraction}

Standardized form was used to extract data from individual studies, for example, author, publication year, study design, 
participants' characteristics, number of participants, treatment duration, intervention, comparators, and outcome measures. Two reviewers extracted data independently (WL, NP). Discrepancies were resolved by a third reviewer (NS).

\section{Quality assessment}

Methodologic quality of each study was assessed using the Cochrane risk of bias tool. ${ }^{12}$ The risk-of-bias domains encompass random sequence generation, allocation concealment, blinding of participants and personnel, blinding of outcome assessment, incomplete outcome data, selective reporting, and other bias. Results of bias were judged as high risk, low risk, or unclear risk. Study quality assessment was performed independently by two investigators (WL, NP). Inconsistencies were resolved by a third reviewer (NS).

\section{Statistical analyses}

Meta-analysis was conducted for SBP, DBP, TC, TG, LDL, HDL, FPG, HOMA-IR, and BW. Treatment effect was estimated with mean difference (MD) in the final assessment between kiwifruit and control groups. The inverse-varianceweighted method was used for pooling MD and estimating 95\% CI. When mean or SDs were not available, they were computed from the available statistical data using appropriate formula. ${ }^{13,14} \mathrm{Q}$ statistic was used to assess the existence of heterogeneity and the cutoff of significant level was $P<0.1$. If heterogeneity was nonsignificant, the fixed-effects model was used, otherwise the random-effects model was used. $I^{2}$ statistic was used to quantify the degree of heterogeneity. If $I^{2}$ values were $50 \%$ or more, then substantial heterogeneity was considered. Subgroup analysis included limiting to studies in hypertensive patients, whole kiwifruit, and treatment duration of at least 8 weeks, when data available. Analysis of data was undertaken with Review Manager Software (RevMan 5.3.5). We planned to detect reporting bias by funnel plot and Egger's test. ${ }^{15}$ However, as the number of studies included in each meta-analysis was small, funnel plot and Egger's test were eventually not performed.

\section{Results}

\section{Search results and study characteristics}

Figure 1 illustrates the procedure of studies selection. A total of 642 citations were initially identified. Three hundred forty-four records were screened after duplicates were removed. After screening titles and abstracts, 15 fulltext articles were retrieved for eligibility analysis. Three studies ${ }^{9,16,17}$ were excluded for being nonrandomized design.
Four reports enrolled the same patients, but reported different outcomes. ${ }^{18-21}$ Only study that reported outcomes most relevant to our meta-analysis, that is, SBP, DBP, TC, TG, LDL, HDL, FPG, HOMA-IR, and BW, was included..$^{20}$ The remaining three reports were excluded. ${ }^{18,19,21}$ Four studies were further excluded because they enrolled subjects with other conditions, for example, allergic diseases and constipation. ${ }^{22-25}$ Finally, five randomized controlled trials met the inclusion criteria and were included in this systematic review and meta-analysis. ${ }^{20,26-29}$ One study enrolled hypercholesterolemic subjects. ${ }^{20}$ Two trials were conducted in hypertensive patients, ${ }^{26,29}$ one each in T2DM ${ }^{28}$ and male smokers. ${ }^{27}$ Study period ranged from 4 weeks to 9 months. Kiwifruit preparations used in each trial varied widely. Three trials used kiwifruit of $A$. deliciosa, ${ }^{20,27,29}$ and one study each used kiwifruit juice of $A$. chinensis ${ }^{28}$ and kiwifruit capsules. ${ }^{26}$ Four studies practically evaluated kiwifruit vs placebo, ${ }^{20,26-28}$ although in two studies healthy or habitual diet was followed in both the treatment and the control groups. ${ }^{20,27}$ One study assessed kiwifruit against apple. ${ }^{29}$ One trial recruited subjects from New Zealand. ${ }^{20}$ Two trials each were conducted in China ${ }^{26,28}$ and Norway. ${ }^{27,29}$ Four trials were published in English ${ }^{20,27-29}$ and one in Chinese. ${ }^{26}$ The characteristics of the included studies are presented in Table 1.

\section{Risk of bias in the eligible studies}

Two studies mentioned how to generate random number. ${ }^{20,29}$ Only one study described proper allocation concealment, ${ }^{29}$ and the others were of unclear risk of selection bias. ${ }^{20,26-28}$ Blinding of participants and personnel was undertaken in one study. ${ }^{29}$ Two studies $^{20,27}$ were open-label. Blinding of outcome assessors was unclear in all studies. The risk of attrition, reporting, and other biases were considered to be low in all studies. Risk-of-bias graph and summary are shown in Figures 2 and 3.

\section{Effect on blood pressure}

The pooled result from four studies ${ }^{20,26,27,29}$ illustrated that kiwifruit did not affect both SBP and DBP compared to control, with MD of $-1.72 \mathrm{mmHg}$ (95\% CI: -4.27 to 0.84$)$ and $-2.35 \mathrm{mmHg}$ (95\% CI: -5.10 to 0.41$)$, respectively (Figure 4).

Three studies contributed data for subgroup analysis in hypertensive patients. ${ }^{26,27,29}$ Of these three studies, two were exclusively conducted in hypertensive patients, ${ }^{26,29}$ and one included a subgroup of hypertensive patients. ${ }^{27}$ Again, no effect was observed on both SBP $(-3.14 \mathrm{mmHg}, 95 \% \mathrm{CI}$ -7.18 to 0.91$)$ and DBP $(-1.68 \mathrm{mmHg}, 95 \% \mathrm{CI}-4.04$ to 0.67$)$. 


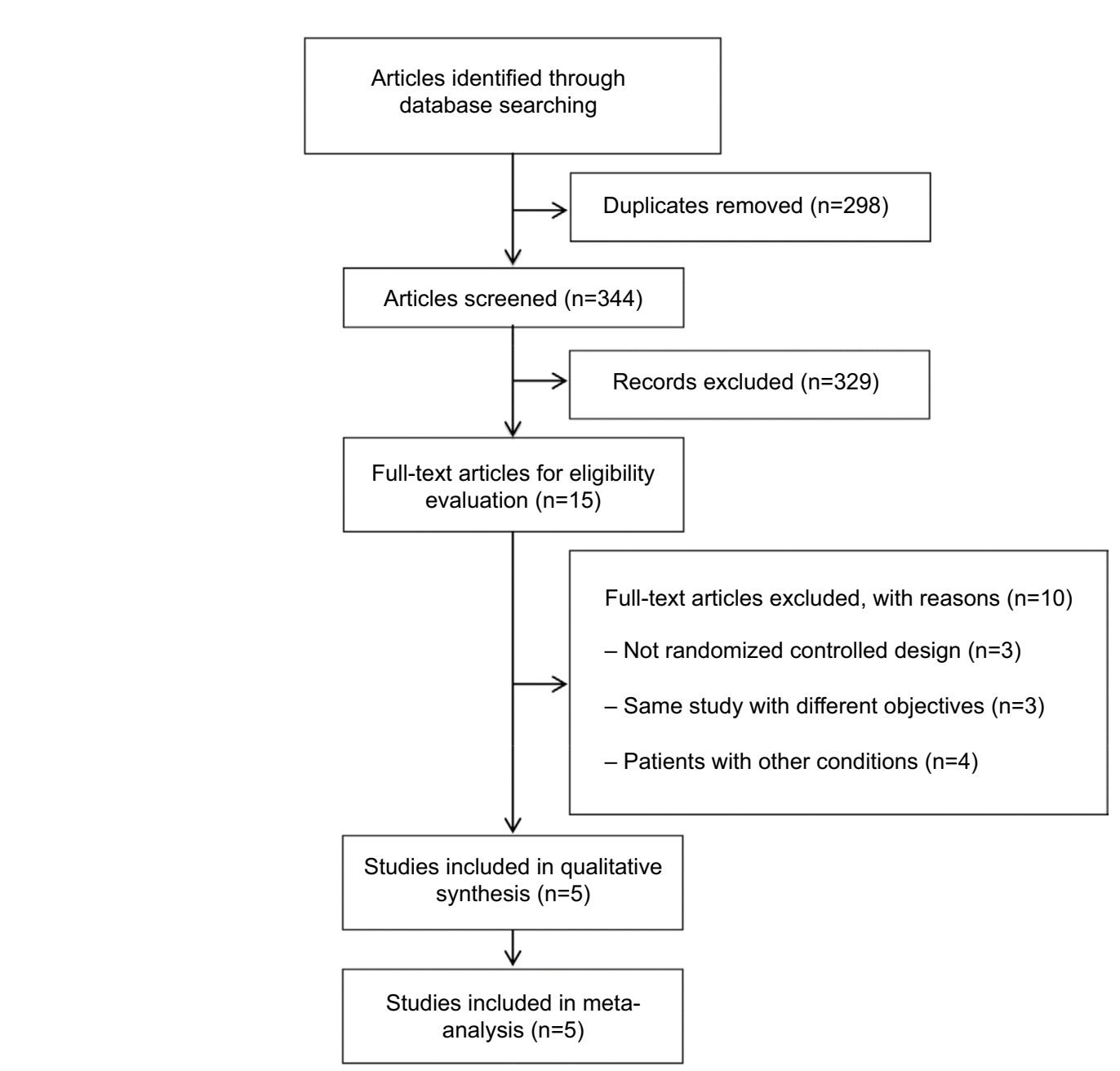

Figure I Flow diagram of studies selection.

\section{Effect on lipid profile}

Four studies were included in the meta-analysis of TC and $\mathrm{TG}^{20,26-28}$ and three studies provided data for the metaanalysis of LDL and HDL. ${ }^{20,27,28}$ No effect of kiwifruit was observed on lipid profile ( $\mathrm{TC}-0.14 \mathrm{mmol} / \mathrm{L}, 95 \% \mathrm{CI}-0.71$ to 0.43 ; TG $-0.23 \mathrm{mmol} / \mathrm{L}, 95 \% \mathrm{CI}-0.66$ to 0.20 ; $\mathrm{LDL}-0.41$ $\mathrm{mmol} / \mathrm{L}, 95 \% \mathrm{CI}-0.99$ to 0.18 ; HDL $0.15 \mathrm{mmol} / \mathrm{L}, 95 \%$ $\mathrm{CI}-0.18$ to 0.48 ) (Figure 5). Subgroup analysis limiting to studies of whole kiwifruit and duration of intervention of at least 8 weeks again revealed no such effect of kiwifruit on TC, TG, LDL, and HDL (Table 2).

\section{Effect on glycemic outcomes}

Pooled data from three trials $\mathrm{s}^{20,26,28}$ showed no significant effect of kiwifruit in reducing FPG. Two studies provided poolable data on HOMA-IR. ${ }^{20,28}$ Kiwifruit exerted no effect on HOMA-IR compared with control (Figure 6). Only one study measured and reported A1C and 2-h PG. ${ }^{28}$ No statistical differences in $\mathrm{A} 1 \mathrm{C}(8.4 \% \pm 1.9 \%$ vs $8.5 \% \pm 1.6 \%)$ and $2-\mathrm{h}$ $\mathrm{PG}(14.0 \pm 3.2 \mathrm{mmol} / \mathrm{L}$ vs $13.9 \pm 3.4 \mathrm{mmol} / \mathrm{L})$ were detected between the two groups at the end of study period. ${ }^{28}$

\section{Effect on BW}

$\mathrm{BW}$ was reported among three trials..$^{20,28,29} \mathrm{BW}$ did not change with kiwifruit compared to control (MD - $1.08 \mathrm{~kg}$, 95\% CI -4.22 to 2.05 ) (Figure 6C).

\section{Discussion}

Kiwifruit contains abundant antioxidants, such as vitamin C, carotenoids, flavonoids, and phenolic components. These components are protective against CVD. Vanillic acid, skimmetin, isoscopoletin, sitogluside, fraxetin, emodin, (+)-catechin, questin, stearic acid, and quercetin were identified in A. chinensis Planch. ${ }^{30}$ Flavonoids quercetin has been shown to possess angiotensin I-converting enzyme (ACE) inhibition activity. ${ }^{31}$ In addition, aqueous and $70 \%$ ethanol extracts of 


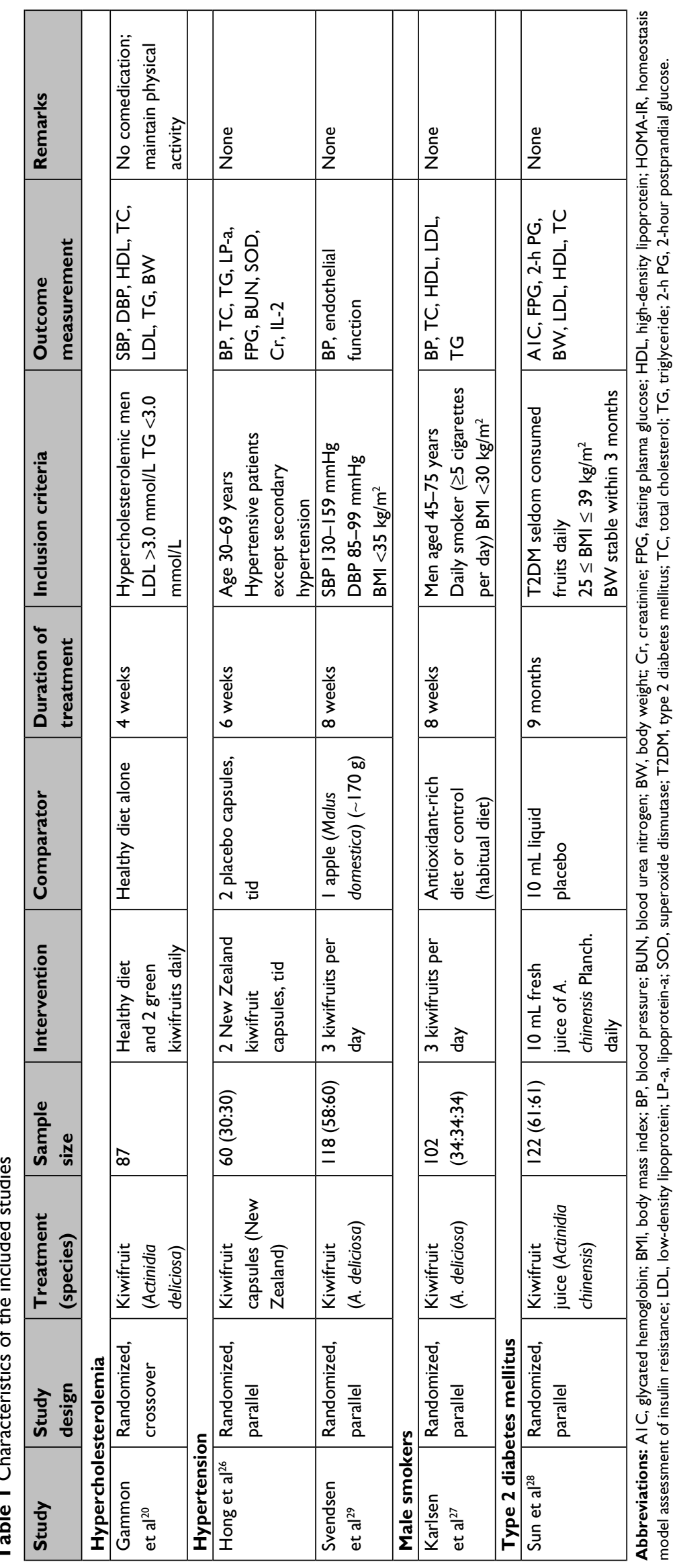




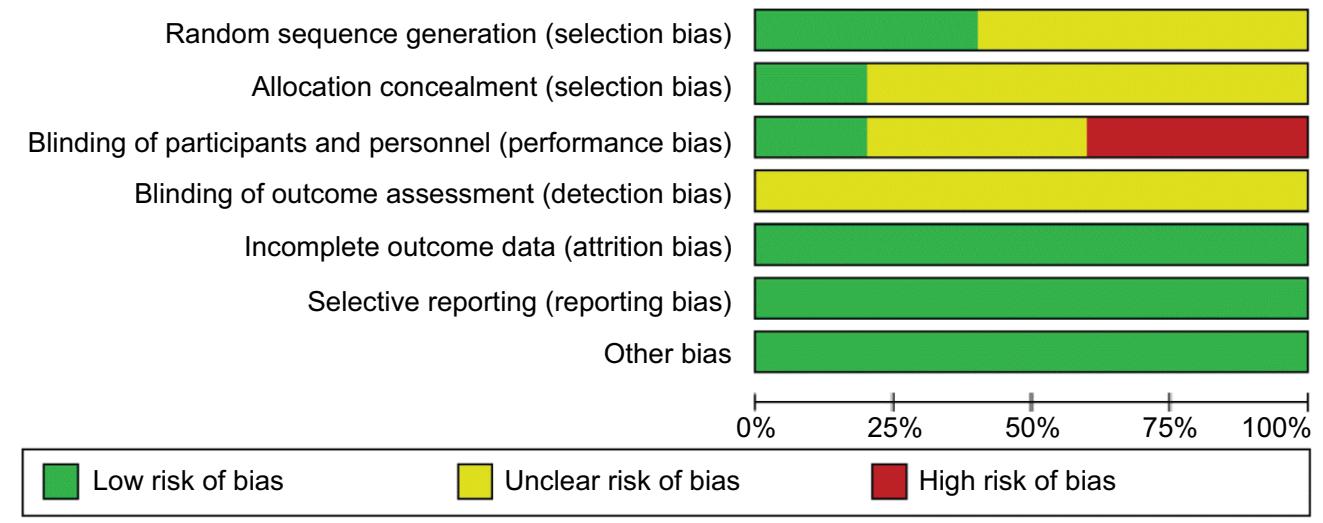

Figure 2 Risk-of-bias graph.
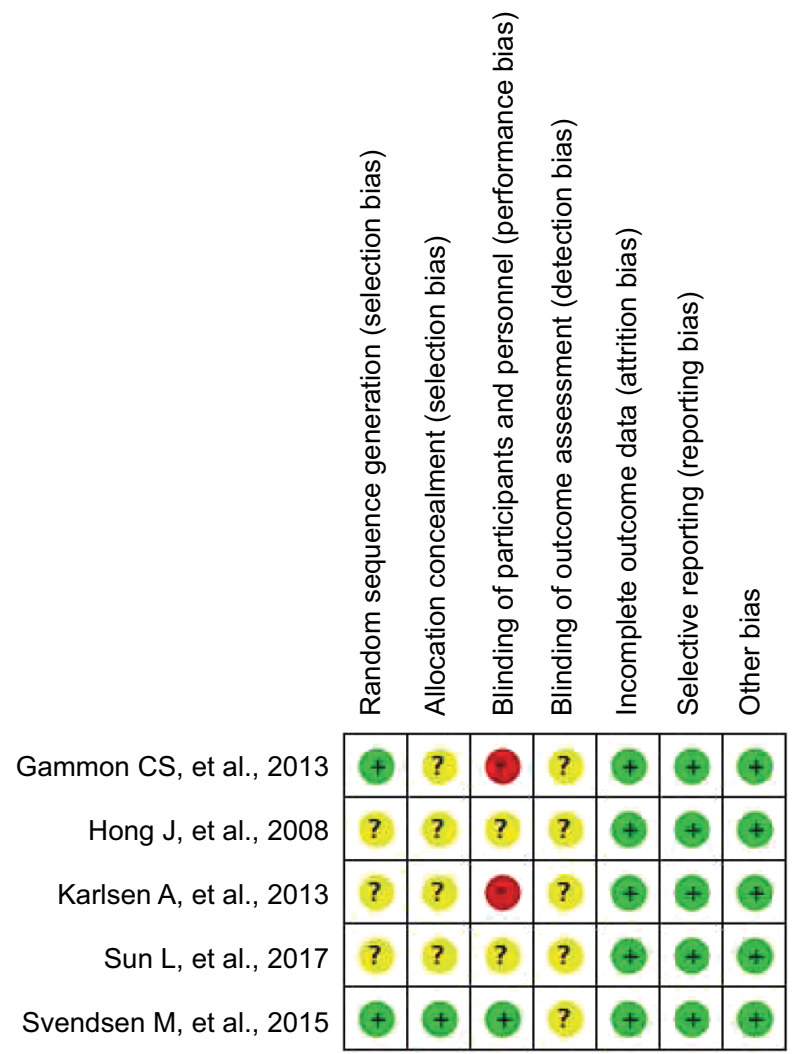

Figure 3 Risk-of-bias summary.

Note: Red (-): high risk of bias; yellow (?): unclear risk of bias; green $(+)$ : low risk of bias.

kiwifruit provided antioxidant and fibrinolytic effects and inhibitory activities against ACE, and HMG-CoA reductase in vitro. ${ }^{32}$ Aqueous extract of kiwifruit was also reported to inhibit both human platelet aggregation and plasma ACE activity in a dose-dependent manner. Inhibition of platelet aggregation was mediated partly by reducing thromboxane $\mathrm{A}_{2}$ synthesis. ${ }^{33}$ Based on these activities, kiwifruit has potential cardiovascular protective properties.
Phenolic components and flavonoid isoquercitrin and quercetin may play a role in lowering blood glucose. ${ }^{8,34}$ Isoquercitrin inhibits $\alpha$-glucosidase and glucose 6-phosphatase. It has potency similar to that of 1-deoxynojirimycin, an $\alpha$-glucosidase inhibitor. ${ }^{34}$ Quercetin possibly demonstrates positive effect in diabetes through attenuating oxidative stress and maintaining pancreatic $\beta$-cell integrity. ${ }^{35}$ It also stimulates insulin secretion by direct activation of L-type $\mathrm{Ca}^{2+}$ channels to increase $\left[\mathrm{Ca}^{2+}\right]$ ion. ${ }^{36}$ Such hypoglycemic effect has been demonstrated in alloxan-induced diabetic rats in which A. deliciosa extract reduced blood glucose level significantly compared to diabetic control group. ${ }^{8}$

Kiwifruit possesses antioxidative effect, removing ROS and preventing formation of ROS, lipid peroxidation of cell membrane, and LDL oxidation. It promotes nitric oxide production and inhibits inflammation and platelet aggregation. A. deliciosa "Hayward" was found to decrease TG, TC, and LDL-C in rats. ${ }^{37}$ Dietary fiber in kiwifruit, especially soluble fiber, may lower cholesterol and TGs in the blood by binding exogenous cholesterol, reducing its absorption, increasing excretion in feces, lowering reabsorption of fatty acids, and decreasing LDL-C formation. ${ }^{37}$ These effects may be helpful for dyslipidemic patients. In addition, kiwifruit was reported to have beneficial effects in patients with constipation problem ${ }^{25}$ and neuropathic diabetic foot ulcer. ${ }^{38}$ One study, being a nonrandomized trial and therefore not included in our systematic review, demonstrated that consumption of two kiwifruits per day for 8 weeks could improve HDL and decrease LDL/ HDL ratio and TC/HDL ratio in hyperlipidemic subjects. ${ }^{9}$

Our meta-analysis showed that kiwifruit had no effect on metabolic health, as measured by SBP, DBP, TC, TG, LDL, HDL, FPG, HOMA-IR, and BW, in participants with cardiovascular risk factors including hypercholesterolemia, hypertension, T2DM, and smokers. Subgroup analysis in 
A

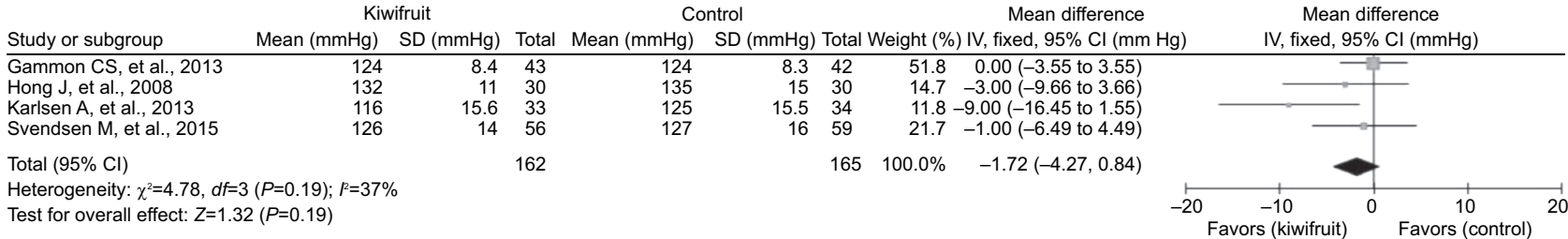

B

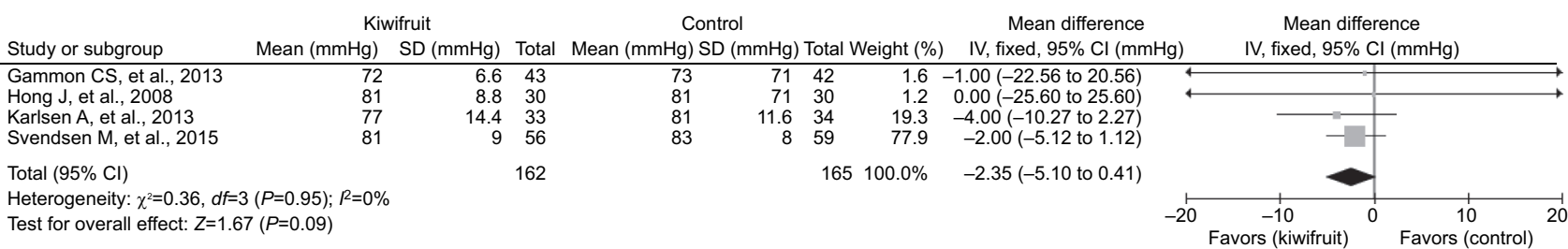

Figure 4 Forest plots of the effect of kiwifruit on $\operatorname{SBP}(\mathbf{A})$ and $\mathrm{DBP}(\mathbf{B})$.

A

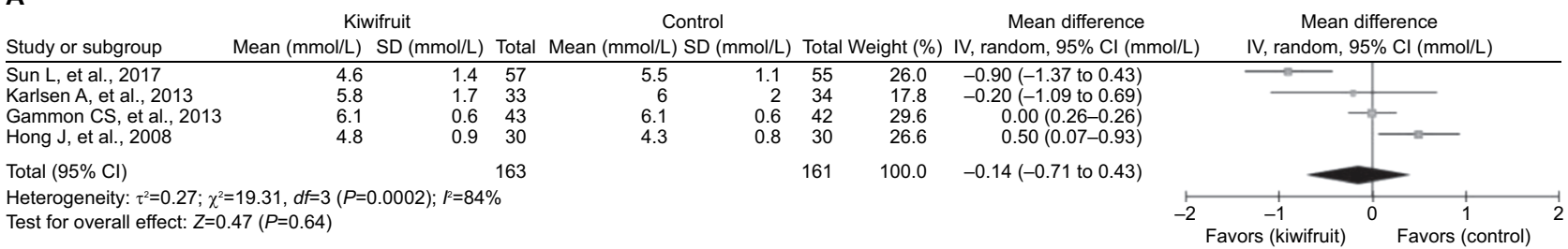

B

Kiwifruit Control Mean difference difference

Study or subgroup Mean (mmol/L) SD (mmol/L) Total Mean (mmol/L) SD (mmol/L) Total Weight (\%) IV, random, $95 \% \mathrm{Cl}(\mathrm{mmol} / \mathrm{L}) \quad \mathrm{IV}, \mathrm{random}, 95 \% \mathrm{Cl}(\mathrm{mmol} / \mathrm{L})$ \begin{tabular}{lllllllll}
\hline Gammon CS, et al., 2013 & 1.6 & 0.5 & 43 & 1.6 & 0.4 & 42 & 34.3 & $0.00(-0.19$ to 0.19$)$
\end{tabular} $\begin{array}{lrrrrrrrr}\text { Hong J, et al., 2008 } & 2 & 1.5 & 30 & 1.9 & 1.1 & 30 & 19.5 & 0.10(-0.57 \text { to } 0.77) \\ \text { Karlsen A, et al., 2013 } & 1.3 & 1.2 & 33 & 1.5 & 1.5 & 34 & 19.9 & -0.20(-0.85 \text { to } 0.45)\end{array}$ $\begin{array}{lllllllrr}\text { Karlsen A, et al., 2013 } & 1.3 & 1.2 & 33 & 1.5 & 1.5 & 34 & 19.9 & -0.20(-0.85 \text { to } 0.45) \\ \text { Sun L, et al., 2017 } & 1.8 & 1.1 & 57 & 2.6 & 1.3 & 55 & 26.3 & -0.80(-1.25 \text { to }-0.35)\end{array}$

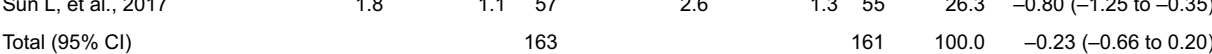

Heterogeneity: $\tau^{2}=0.13 ; \chi^{2}=10.87, d f=3(P=0.01) ; P^{2}=72 \%$ Test for overall effect: $Z=1.05(P=0.29)$

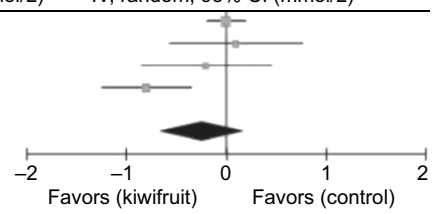

C

Kiwifruit Control Mean difference difference Study or subgroup $\quad$ Mean ( $\mathrm{mmol} / \mathrm{L}) \quad \mathrm{SD}(\mathrm{mmol} / \mathrm{L})$ Total Mean (mmol/L) SD (mmol/L) Total Weight (\%) IV, random, $95 \% \mathrm{Cl}(\mathrm{mmol} / \mathrm{L}) \quad \mathrm{IV}, \mathrm{random}, 95 \% \mathrm{Cl}(\mathrm{mmol} / \mathrm{L})$ \begin{tabular}{lllllllll}
\hline Gammon CS, et al., 2013 & 3.9 & 0.5 & 43 & 4 & 0.5 & 42 & 41.1 & $-0.10(-0.31$ to 0.11$)$
\end{tabular} $\begin{array}{lllllllll}\text { Sun L, et al., 2017 } & 3.7 & 1.5 & 33 & 3.9 & 1.9 & 34 & 23.5 & -0.20(-1.02 \text { to } 0.62)\end{array}$

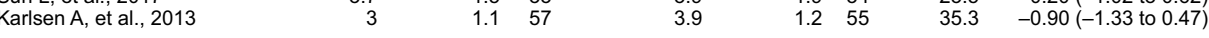

Total $(95 \% \mathrm{Cl})$ 133

Heterogeneity: $\tau^{2}=0.21 ; \chi^{2}=10.83, d f=2(P=0.004) ; k=82 \%$

Test for overall effect: $Z=1.36(P=0.17)$

$131 \quad 100.0 \quad-0.41(-0.99$ to 0.18$)$

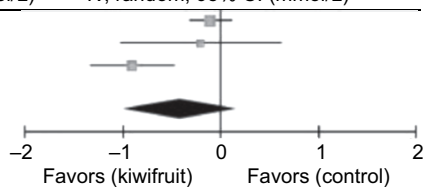

D

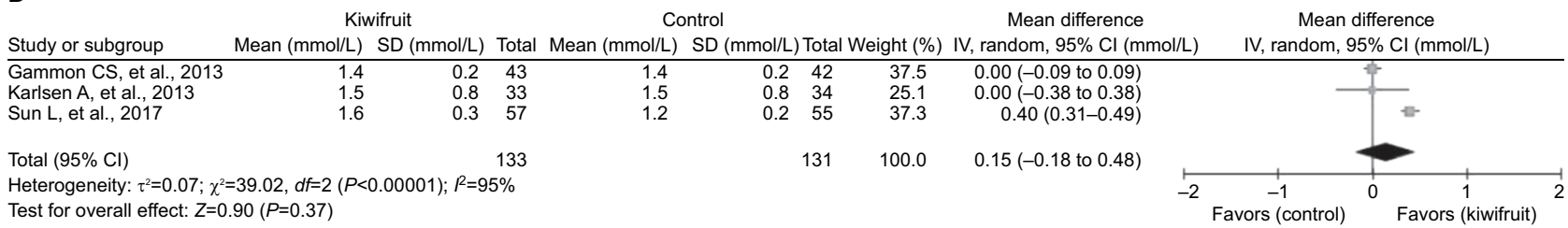

Figure 5 Forest plots of the effect of kiwifruit on total cholesterol (A), triglyceride (B), low-density lipoprotein (C), and high-density lipoprotein (D).

hypertensive patients also suggested no effect of kiwifruit on blood pressure. Similarly, using kiwifruit intervention for at least 8 weeks or using whole fruit also did not affect lipid profile. It is noted that various dosages and forms of kiwifruit were utilized including whole fruit, kiwifruit juice, and kiwifruit extract capsules. Although we included the participants with cardiovascular risk factors, a wide variety of participants were involved. For example, one study recruited 
Table 2 Meta-analysis of effect of kiwifruit on lipid profile stratified by subgroups

\begin{tabular}{|c|c|c|c|c|c|c|c|c|c|}
\hline \multirow[t]{2}{*}{ Outcome } & \multirow[t]{2}{*}{ Subgroup } & \multirow{2}{*}{$\begin{array}{l}\text { No. of } \\
\text { studies }\end{array}$} & \multirow{2}{*}{$\begin{array}{l}\text { No. of } \\
\text { patients }\end{array}$} & \multirow{2}{*}{$\begin{array}{l}\text { Effects } \\
\text { model }\end{array}$} & \multirow[t]{2}{*}{ MD } & \multirow[t]{2}{*}{$95 \% \mathrm{Cl}$} & \multicolumn{2}{|c|}{ Heterogeneity } & \multirow[t]{2}{*}{$P$-value } \\
\hline & & & & & & & $P$-value & $I^{2}(\%)$ & \\
\hline \multirow[t]{3}{*}{ TC } & Overall & 4 & 324 & Random & -0.14 & $-0.7 I$ to 0.43 & 0.0002 & 84 & 0.64 \\
\hline & Intervention duration $\geq 8$ weeks & 3 & 264 & Random & -0.37 & -1.02 to 0.29 & 0.004 & 82 & 0.29 \\
\hline & Whole kiwifruit & 2 & 152 & Fixed & -0.02 & -0.26 to 0.23 & 0.67 & 0 & 0.90 \\
\hline \multirow[t]{3}{*}{ TG } & Overall & 4 & 324 & Random & -0.23 & -0.66 to 0.20 & 0.01 & 72 & 0.29 \\
\hline & Intervention duration $\geq 8$ weeks & 3 & 264 & Random & -0.32 & -0.86 to 0.22 & 0.005 & 81 & 0.25 \\
\hline & Whole kiwifruit & 2 & 152 & Fixed & -0.02 & -0.20 to 0.17 & 0.56 & 0 & 0.86 \\
\hline \multirow[t]{3}{*}{ LDL } & Overall & 3 & 264 & Random & -0.41 & -0.99 to 0.18 & 0.004 & 82 & 0.17 \\
\hline & Intervention duration $\geq 8$ weeks & 3 & 264 & Random & -0.41 & -0.99 to 0.18 & 0.004 & 82 & 0.17 \\
\hline & Whole kiwifruit & 2 & 152 & Fixed & -0.11 & -0.31 to 0.10 & 0.82 & 0 & 0.31 \\
\hline \multirow[t]{3}{*}{ HDL } & Overall & 3 & 264 & Random & 0.15 & -0.18 to 0.48 & $<0.0000$ I & 95 & 0.37 \\
\hline & Intervention duration $\geq 8$ weeks & 3 & 264 & Random & 0.15 & -0.18 to 0.48 & $<0.00001$ & 95 & 0.37 \\
\hline & Whole kiwifruit & 2 & 152 & Fixed & 0.00 & -0.08 to 0.08 & 1 & 0 & 1 \\
\hline
\end{tabular}

Abbreviations: HDL, high-density lipoprotein; LDL, low-density lipoprotein; TC, total cholesterol; TG, triglyceride.

A

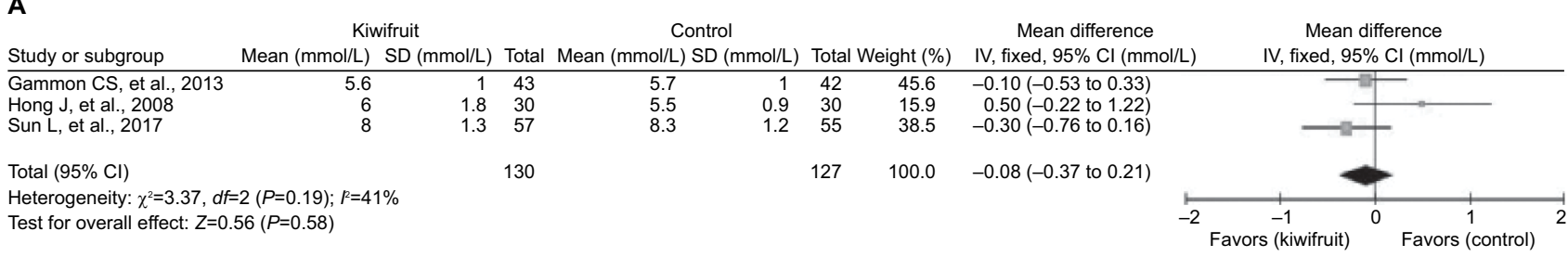

B

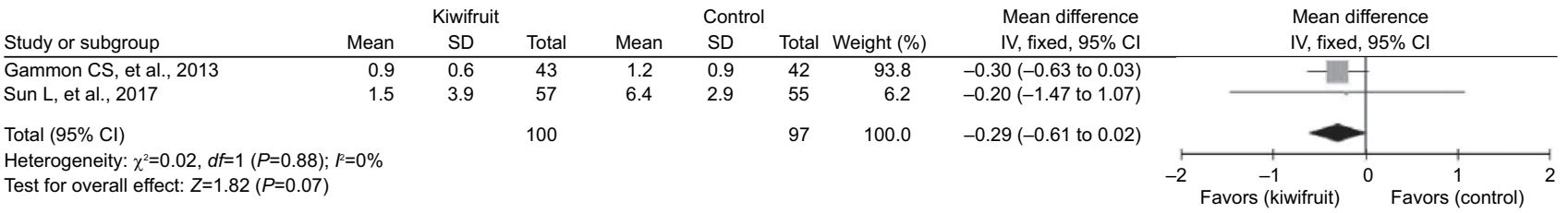

C

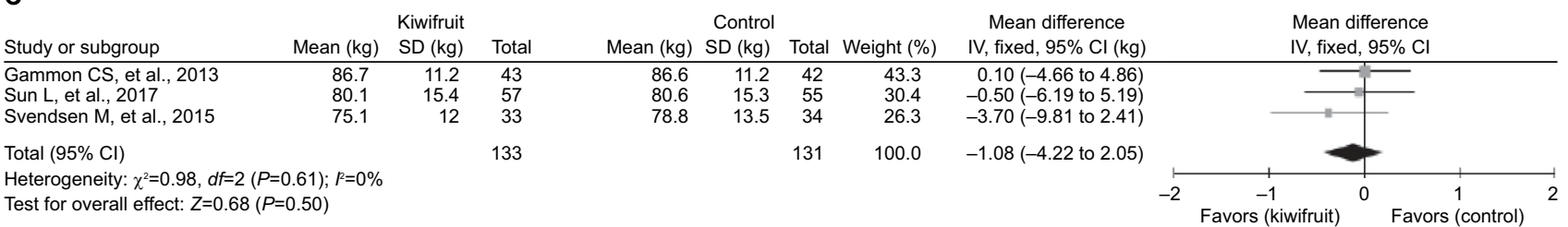

Figure 6 Forest plots of the effect of kiwifruit on fasting plasma glucose (A), homeostasis model assessment of insulin resistance (B), and body weight (C).

hypercholesterolemic men, ${ }^{20}$ and the other enrolled male smokers. ${ }^{27}$ In addition, in one study kiwifruit was added to healthy diet compared to healthy diet alone and patients were instructed to maintain their physical activities. ${ }^{20}$ Therefore, it was difficult to interpret results and to elucidate the sole effects of kiwifruit on these parameters.

A significant heterogeneity was detected in meta-analysis of lipid profile. As described earlier, a wide range of kiwifruit preparations and dosages were used. The species of kiwifruit also differed, namely $A$. deliciosa, ${ }^{20,27,29}$ and $A$. chinensis. ${ }^{28}$
Specific species was not described in the study that used kiwifruit capsules. ${ }^{26}$ The control group also varied, being placebo $^{20,26-28}$ and apple. ${ }^{29}$ Although the participants with cardiovascular risk factors were considered, they differed clinically. And this may contribute to statistical heterogeneity. Other limitations of the included trials are noteworthy. First, most of them enrolled a small number of participants and may lack statistical power. Second, the methodologic quality varied. All of them were of unclear risk of detection bias. Only one trial mentioned the blinding of participants 
and study personnel. ${ }^{29}$ Two studies were nonblinded, ${ }^{20,27}$ and thus may be prone to performance bias.

To our knowledge, this is the first systematic review and meta-analysis of kiwifruit on metabolic health. However, our study is not without limitations. First, significant heterogeneity existed among the results of the studies included as mentioned earlier. Second, only published trials were included, and publication bias cannot be ruled out although we considered both English and non-English publications. Funnel plot and its asymmetry were not evaluated because of a small number of studies included in each meta-analysis. Third, only five trials were included in the systematic review, and even smaller number of studies for meta-analysis of each outcome. Together with the small sample size in each trial, the results of meta-analysis may be imprecise. Additionally, we focused only on major risk factors, namely hypertension, hypercholesterolemia, T2DM, and smoking. Several factors, for example, obesity, physical inactivity, and other unmodifiable risk factors, such as increased age, male gender, and familial history of CVD, were not considered. Obesity and sedentary lifestyle have been known to increase the risk of CVD and metabolic disorders.

\section{Conclusion}

Our meta-analysis suggested a lack of effect of kiwifruit on metabolic health in participants with cardiovascular risk factors, although there was a trend toward the reduction in BP, TC, TG, LDL, FPG, HOMA-IR, and BW and improving HDL. Due to limited evidence and high heterogeneity of the study results, the potential of kiwifruit as a nonpharmaceutical alternative for metabolic health should be further evaluated in well-defined, well-controlled trials with larger sample size and standardized preparation.

\section{Disclosure}

The authors report no conflicts of interest in this work.

\section{References}

1. Kaur J. A comprehensive review on metabolic syndrome. Cardiol Res Pract. 2014;2014(7):1-21.

2. Timmis A, Townsend N, Gale C, et al. European society of cardiology: cardiovascular disease statistics 2017. Eur Heart J. 2018;39(7): 508-579.

3. Expert Panel on Integrated Guidelines for Cardiovascular Health and Risk Reduction in Children and Adolescents; National Heart, Lung, and Blood Institute. Pediatrics. 2011;128(Suppl 5): S213-S256.

4. Whelton PK, Carey RM, Aronow WS, et al. 2017 ACC/AHA/AAPA/ ABC/ACPM/AGS/APhA/ASH/ASPC/NMA/PCNA Guideline for the Prevention, Detection, Evaluation, and Management of High Blood Pressure in Adults: A Report of the American College of Cardiology/ American Heart Association Task Force on Clinical Practice Guidelines. J Am Coll Cardiol. 2018;71(19):e127-e248.
5. Shastri KV, Bhatia V, Parikh PR, Chaphekar VN. Actinidia deliciosa: a review. Int J Pham Sci Res. 2012;3:3543-3549.

6. Ferguson AR. Kiwifruit: a botanical review. In: Janick J, editor. Horticultural Reviews. Hoboken, NJ: John Wiley \& Sons, Inc.; 1984:1-64.

7. Ferguson AR. New temperate fruits-Actinidia chinensis and Actinidia deliciosa. In: Janick J, editor. Perspectives on New Crops and New Uses. Alexandria, VA: ASHS Press; 1999:342-347.

8. Soren G, Sarita MTP. Antidiabetic activity of Actinidia deliciosa fruit in alloxan induced diabetic rats. Pharma Innovation J. 2016;5:31-34.

9. Chang WH, Liu JF. Effects of kiwifruit consumption on serum lipid profiles and antioxidative status in hyperlipidemic subjects. Int J Food Sci Nutr. 2009;60(8):709-716.

10. Duttaroy AK, Jørgensen A. Effects of kiwi fruit consumption on platelet aggregation and plasma lipids in healthy human volunteers. Platelets. 2004;15(5):287-292.

11. Moher D, Liberati A, Tetzlaff J, Altman DG; PRISMA Group. Preferred reporting items for systematic reviews and meta-analyses: the PRISMA Statement. Ann Intern Med. 2009;151(4):264-269.

12. Higgins JP, Altman DG, Gøtzsche PC, et al. The Cochrane Collaboration's tool for assessing risk of bias in randomised trials. BMJ. 2011;343:d5928.

13. Higgins JP, Green S, editors. Cochrane Handbookfor Systematic Reviews of Interventions Version 5.1.0 [updated March 2011]. The Cochrane Collaboration; 2011. Available from www.handbook.cochrane.org.

14. Hozo SP, Djulbegovic B, Hozo I. Estimating the mean and variance from the median, range, and the size of a sample. BMC Med Res Methodol. 2005;5(1):13.

15. Egger M, Davey Smith G, Schneider M, Minder C. Bias in meta-analysis detected by a simple, graphical test. BMJ. 1997;315(7109):629-634.

16. He S. Syrup of Actinidia chinensis treatment for 40 hyperlipidemia. $J$ Tradit Chin Med. 2008;24:152.

17. Zhu L, Zhang K, Meng X. Clinical research on kiwi essence's function of reducing lipemia. Acta Chin Med Pharmacol. 2002;30:12-13.

18. Gammon CS, Kruger R, Brown SJ, Conlon CA, von Hurst PR, Stonehouse W. Daily kiwifruit consumption did not improve blood pressure and markers of cardiovascular function in men with hypercholesterolemia. Nutr Res. 2014;34(3):235-240.

19. Gammon CS, Kruger R, Conlon CA, von Hurst PR, Jones B, Stonehouse W. Inflammatory status modulates plasma lipid and inflammatory marker responses to kiwifruit consumption in hypercholesterolaemic men. Nutr Metab Cardiovasc Dis. 2014;24(1):91-99.

20. Gammon CS, Kruger R, Minihane AM, Conlon CA, von Hurst PR, Stonehouse W. Kiwifruit consumption favourably affects plasma lipids in a randomised controlled trial in hypercholesterolaemic men. $\mathrm{Br} J$ Nutr. 2013;109(12):2208-2218.

21. Gammon CS, Minihane AM, Kruger R, et al. TaqIB polymorphism in the cholesteryl ester transfer protein (CETP) gene influences lipid responses to the consumption of kiwifruit in hypercholesterolaemic men. Br J Nutr. 2014;111(6):1077-1084.

22. Kim SH, Kim S, Lee SH, et al. The effects of PG102, a water-soluble extract from Actinidia arguta, on serum total IgE levels: a double-blind, randomized, placebo-controlled exploratory clinical study. Eur J Nutr. 2011;50(7):523-529.

23. Mishra S, Edwards H, Hedderley D, Podd J, Monro J. Kiwifruit nonsugar components reduce glycaemic response to co-ingested cereal in humans. Nutrients. 2017;9(11)E1195.

24. Mishra S, Willis J, Ansell J, Monro JA. Equicarbohydrate partial exchange of kiwifruit for wheaten cereal reduces postprandial glycaemia without decreasing satiety. J Nutr Sci. 2016;5:e37.

25. Udani JK, Bloom DW. Effects of Kivia powder on gut health in patients with occasional constipation: a randomized, double-blind, placebocontrolled study. Nutr J. 2013;12(1):78.

26. Hong J, Hu J, Li N. Effects of New Zealand kiwifruit capsules on level of SOD in patients with primary hypertension. Chin Prev Med. 2008;9:413-416.

27. Karlsen A, Svendsen M, Seljeflot I, et al. Kiwifruit decreases blood pressure and whole-blood platelet aggregation in male smokers. J Hum Hypertens. 2013;27(2):126-130. 
28. Sun L, Li X, Li G, Dai B, Tan W. Actinidia chinensis Planch. Improves the indices of antioxidant and anti-inflammation status of type 2 diabetes mellitus by activating Keap1 and Nrf2 via the upregulation of microRNA-424. Oxid Med Cell Longev. 2017;2017:7038789.

29. Svendsen M, Tonstad S, Heggen E, et al. The effect of kiwifruit consumption on blood pressure in subjects with moderately elevated blood pressure: a randomized, controlled study. Blood Press. 2015;24(1):48-54.

30. Ru J, Li P, Wang J, et al. TCMSP: a database of systems pharmacology for drug discovery from herbal medicines. J Cheminform. 2014;6(1):13.

31. Guerrero L, Castillo J, Quiñones M, et al. Inhibition of angiotensinconverting enzyme activity by flavonoids: structure-activity relationship studies. PLoS One. 2012;7(11):e49493.

32. Jung KA, Song TC, Han D, Kim IH, Kim YE, Lee CH. Cardiovascular protective properties of kiwifruit extracts in vitro. Biol Pharm Bull. 2005;28(9):1782-1785.

33. Dizdarevic LL, Biswas D, Uddin MD, et al. Inhibitory effects of kiwifruit extract on human platelet aggregation and plasma angiotensinconverting enzyme activity. Platelets. 2014;25(8):567-575.
34. Kurakane S, Yamada N, Sato H, Igarashi K. Anti-diabetic effects of Actinidia arguta polyphenols on rats and KK-Ay mice. Food Sci Technol Res. 2011;17(2):93-102.

35. Coskun O, Kanter M, Korkmaz A, Oter S, Quercetin OS. Quercetin, a flavonoid antioxidant, prevents and protects streptozotocin-induced oxidative stress and beta-cell damage in rat pancreas. Pharmacol Res. 2005;51(2):117-123.

36. Bardy G, Virsolvy A, Quignard JF, et al. Quercetin induces insulin secretion by direct activation of L-type calcium channels in pancreatic beta cells. Br J Pharmacol. 2013;169(5):1102-1113.

37. Leontowicz M, Jesion I, Leontowicz H, et al. Health-promoting effects of ethylene-treated kiwifruit 'hayward' from conventional and organic crops in rats fed an atherogenic diet. J Agric Food Chem. 2013;61(15):3661-3668.

38. Mohajeri G, Safaee M, Sanei MH. Effects of topical kiwifruit on healing of neuropathic diabetic foot ulcer. J Res Med Sci. 2014;19(6): $520-524$.
Diabetes, Metabolic Syndrome and Obesity: Targets and Therapy is an international, peer-reviewed open-access journal committed to the rapid publication of the latest laboratory and clinical findings in the fields of diabetes, metabolic syndrome and obesity research. Original research, review, case reports, hypothesis formation, expert opinion and commentaries are all considered for publication. The manuscript management system is completely online and includes a very quick and fair peer-review system, which is all easy to use. Visit http://www.dovepress.com/testimonials.php to read real quotes from published authors. 